\title{
Le système des tours de parole dans Un été de canicule de Françoise Bourdin
}

\author{
Dr. Noha Salah El Din Awad \\ Professeur adjoint (linguistique) \\ Faculté des Lettres-Université de \\ Ménoufeya
}




\section{résumé \\ Le système des tours de parole dans Un été de canicule de Françoise Bourdin}

Parler c'est communiquer avec les autres,s'exprimer,se défendre ou s'entendre.En un mot, parler c'est s'affirmer en tant qu'individu social.

Souscrivant à cette importance fonctionnelle de la parole,les écrivains ont recours aux différentes formes d'interactions verbales dans leurs oeuvres. Le fonctionnement de ces interactions obéit à des règles qui gèrent,en premier lieu,le passage des tours de parole entre les interlocuteurs.Ces tours s'organisent dans un système qui illustre leur parcours opérationnel.

Notre étude se focalise sur l'analyse du système des tours de parole dans Un été de canicule de Françoise Bourdin.Ce roman raconte l'histoire des Soubeyrand torturés par un secret qui risque de détruire leur vie.Les différents échanges langagiers des Soubeyrand sont abordés à la lumière des deux questions soulevées par le système des tours:l'emplacement du changement de tour,et la nature du successeur.L'analyse de ces questions s'effectue sur la base des propriétés descriptives des tours qui soulignent la synchronisation interactionnelle.

Bref,avec Un été de canicule,le système des tours de parole gagne de l'ampleur grâce à la mise en relief des règles conversationnelles qui garantissent la poursuite de l'enchaînement discursif.

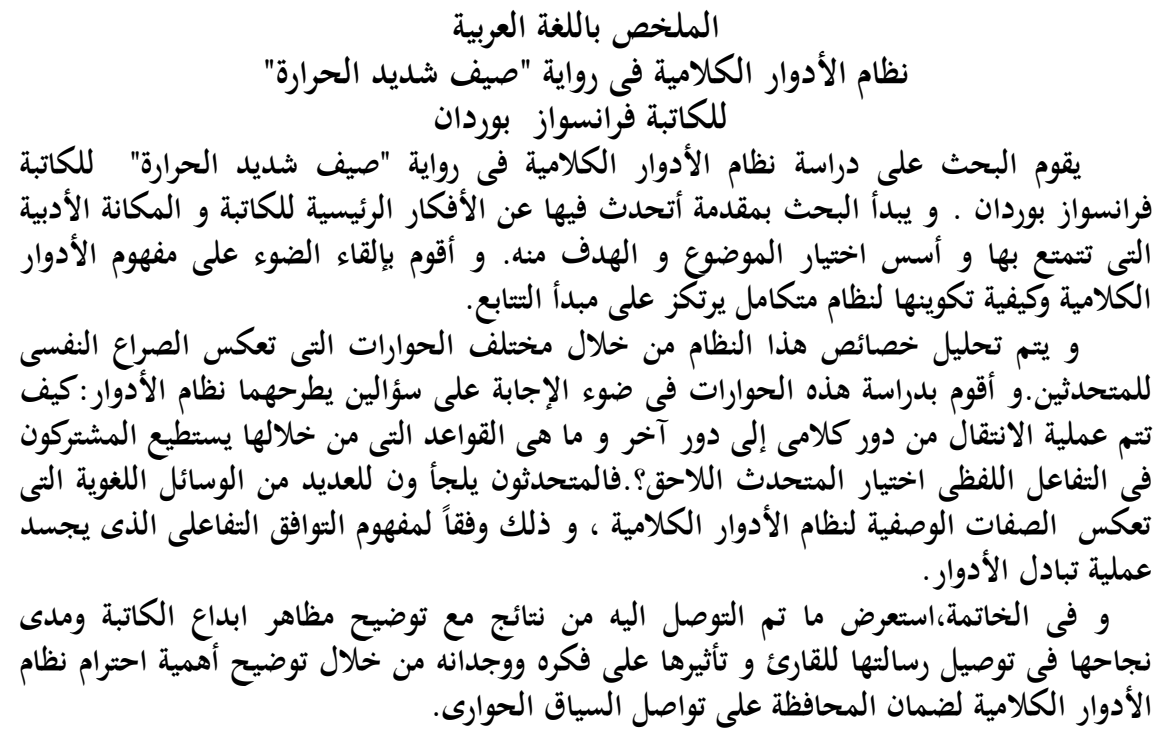


Romancière française et scénariste pour la

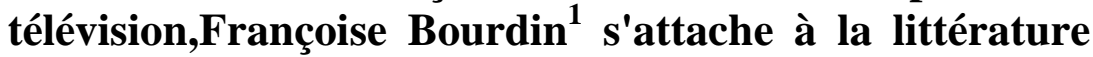
dès son enfance.Son premier roman Les Soleils mouillés $^{2}$ est paru chez Julliard à l'âge de vingt ans.Après plusieurs années consacrées à sa famille, elle revient à l'écriture et offre aux lecteurs une quarantaine de romans publiés pour la plupart chez Belfond,depuis 1994.

À travers ces romans à succès dont Terre indigo $^{3}$,série phare de TF1,Bourdin parvient à tenir en haleine un public rassemblé davantage autour de son univers romanesque qui"prend racine dans les histoires de famille,les secrets et les passions qui les traversent". ${ }^{\prime 4}$ pes personnages dépeints avec soin touchent au vif les lecteurs qui partagent aussi bien leur enthousiasme que leurs déchirements.Ce qui assure à Bourdin une place distinguée parmi auteurs les plus lus en France" ${ }^{\prime 5}$.

C'est surtout avec Un été de canicule 6 que Bourdin s'approche au plus près de ses lecteurs grâce à des personnages qu'elle fait revivre à travers de multiples conversations ${ }^{7}$.Ce roman, qui nous a vivement intéressée, invite à la découverte du secret qui fonde la trame de l'histoire des Soubeyrand.À leur tête,Emma Soubeyrand, la patronne du Café des Tilleuls à Cucuron,se dévoue à ses quatre enfants.Antoine,l'aîné des Soubeyrand,est parti pour le Brésil après la disparition de Labaume ${ }^{8}$ depuis sept ans, Paul ,le pépiniériste, vient de se marier à Marine,Vincent est coiffeur et Sophi e avocate. Malheureusement,Vincent devient victime d'un terrible accident de moto. Ce coup du sort bouleverse la vie des Soubeyrand dont les interactions verbales jalonnent Un été de canicule: 
"À un premier niveau d'analyse',que l'on peut dire "formel",toute interaction(verbale) se présente comme une succession de "tours de parole" "10.

C'est donc sur les tours de parole que repose le mécanisme opérationnel des interactions verbales voire des échanges langagiers susceptibles de confirmer les liens sociaux entre les différents individus.

D'où l'importance accordée à l'analyse structurale des tours de parole:

"ce terme désignant d'abord le mécanisme d'alternance des prises de parole,puis par métonymie,la contribution verbale d'un locuteur déterminé à un moment déterminé du déroulement de l'interaction(...)."11

De la sorte,le principe d'alternance se trouve à la base des tours qui constituent tout" un système de droits et de devoirs ${ }^{12}$ tels que:

1-le locuteur en place $\left(L_{1}:\right.$ "current speaker"13) a le droit de garder la parole un certain temps,mais aussi le devoir de la céder à un moment donné;

2-son successeur potentiel $\left(\mathrm{L}_{2}:\right.$ "next speaker") a le devoir de laisser parler $L_{1}$ et de l'écouter pendant qu'il parle;il a aussi le droit de réclamer la parole au bout d'un certain temps, et le devoir de la prendre quand $L_{1}$ la lui cède" ${ }^{14}$.

Nous allons donc avoir pour objectif de mettre l'accent sur les règles qui conditionnent le fonctionnement de ce système,à la lumière des deux problèmes qui en découlent:

"1.À quel moment s'effectue l'opération de relais?(problème de l'emplacement du changement de tour). 
2.À qui le tour?(problème de la nature du "next speaker")."15

\section{Au lendemain de l'accident de Vincent,Marine converse avec Paul:}

"(...)Paul se trouvait devant un dilemme qui le torturait.Assis sur le canapé du salon,(...),il réfléchissait.Devait-il,oui ou non, appeler Antoine?(...).Paul tendit la main vers l'agenda ouvert près de lui,(...).Le numéro d'Antoine était inscrit en haut de la première page du répertoire(...),Paul pouvait joindre Antoine s'il le voulait.Mais le voulait-il?En faisant revenir son frère aîné,il prendrait un risque qu'il n'avait guère envie de courir.(...).De quelle façon réagirait Marine ${ }^{16}$ ?Remise en présence d'Antoine,qu'éprouverait-elle?

Exaspéré,il se reprocha son manque de confiance en elle.Marine l'aimait,elle lui avait donné un fils,ils étaient heureux tous les trois.

T1 ${ }^{17}$-Paul?Je dois aller travailler,c'est l'heure...Avant de prendre mon service,je passerai voir Vincent et je t'appellerai si j'ai des nouvelles. Mais à mon avis, il n'y a rien de changé...Qu'est-ce que tu fais?

Tandis qu'elle se penchait vers lui,il sentait son parfum,un mélange de lavande et de santal dont il raffolait.À l'instant où leurs lèvres se touchèrent,il l'attira à lui,la fit asseoir sur ses genoux.

T2-Je me demande si je ne devrais pas appeler Antoine,avoua-t-il.

La réaction de Marine fut pire que ce qu'il avait redouté.Elle se leva d'un bond,d'abord stupéfaite,puis tout de suite affolée.

T3-Antoine?Pourquoi? 
T4-Si Vincent ne...Si Vincent devait mourir...Antoine voudra être là.

T5-Mais on n'en sait rien!

T6-Tu m'as dit toi-même que les médecins n'étaient pas très optimistes.Antoine est au bout du monde,il ne peut pas revenir en cinq minutes, il faut le prévenir.

Il voyait bien qu'elle aurait voulu protester,argumenter,le dissuader, cependant elle ne trouvait rien.

T7-Fais comme tu veux,concéda-t-elle enfin d'une voix rauque.

Sans plus penser à l'embrasser,elle ramassa son sac,ses clefs, et sortit en hâte,le laissant complètement désemparé. ${ }^{\prime 18}$

"La situation communicative"19 de ce "dilogue" 20 est préétablie par la narratrice"(...)Paul se trouvait...les trois". Ce qui contribue à déterminer les fondateurs des tours de parole qui constituent le schéma participatif illustré par "(...)le nombre des participants impliqués,la distribution des rôles interlocutifs, ainsi que les caractéristiques proxémiques de la communication(...)"

Le format de réception est composé de deux participants ratifiés: Paul et sa femme.Chacun est "le destinataire privilégié" 22 de l'autre.Le format de production est occupé ,en premier lieu, par Marine sur laquelle est focalisé le paragraphe précédant directement le dilogue.Elle fait donc figure de la locutrice qui ouvre l'échange verbal.Son destinataire est dénoté par

"la nomination explicite" de nature verbale ${ }^{24}$ marque "l'allocation du tour à 
quelqu'un de précis"25. Il indique, de ce fait, la nature du successeur dont le caractère évident au cours du dilogue se justifie par:

-La nature intrinsèque du dispositif énonciatif du dilogue "où l'alternance des locuteurs, jointe à la teneur même de leurs propos, permet d'identifier sans trop de problème celui qui parle" ${ }^{\prime 26}$.

-Les divers commentaires ${ }^{27}$ de la narratrice qui,loin d'entraver l'enchaînement des prises de parole, soulignent"les configurations proxémiques et posturales des interlocuteurs ${ }^{28}$ "29,"Tandis...genoux"," La réaction...affolée", ainsi que leur état émotionnel,"Il voyait...rien".Ce qui renseigne le lecteur sur le positionnement réciproque des locuteurs et contribue d'une grande part à l'identification du destinataire.

-Le discours attributif qui,placé à la fin du deuxième et du dernier tours, "avoua-t-il(Paul),concéda-telle(Marine)", "précis(e) quel personnage parle, à qui il s'adresse." ${ }^{30}$

Une fois son successeur déterminé,le locuteur se trouve dans l'obligation de lui céder la parole . Ce changement de tour s'opère à travers plusieurs "signaux de fin de tour" ${ }^{31}$ dont la nature verbale ${ }^{32}$ est mise en relief par le statut illocutoire des énoncés dans le dilogue.

Le premier tour de parole $^{33}$ se termine par une question," Qu'est-ce que tu fais?",considérée comme"(...)un acte (de langage $)^{34}$ initiatif qui généralement sollicite fortement une réaction verbale (ou à la rigueur un substitut gestuel )" ${ }^{35}$.

Paul assure sa prise de parole par "un élément janus" 36 voire "une intervention" ${ }^{37}$, ,Je...Antoine",ayant une double valeur illocutoire: 
-Réactive ${ }^{38}$ :l'intervention de Paul est constituée d'un acte de langage ${ }^{39}$ assertif qui fournit une réponse à la question de sa femme.

-Initiative:cette assertion acquiert la valeur d'une question directe $^{40}$ marquée lexicalement par le verbe performatif "Je me demande".

Au caractère solliciteur de cette interrogation,vient s'ajouter son effet provocateur sur l'allocutaire Marine qui,"affolée", ne tarde pas à enchaîner du tac au tac par un nouvel élément Janus:"Antoine?Pourquoi?". La première question,"Antoine?", est une reprise "diaphonique" 41 "réitérative" ${ }^{42}$ "hétérodéclenchée ${ }^{43}$.Cette reprise a "une fonction idéelle" 44 et correspond,par la suite,à une demande de confirmation suivie d'une autre demande de justification,"Pourquoi?".Paul remplit son devoir interlocutif. Il prend le tour et confirme ses propos à travers une assertion,"Si...être là",qui"(...)appelle une réaction,en même temps qu'elle est une réponse à une question explicite ou implicite(...)" ${ }^{\prime 45}$.

Marine répond à cet appel par une "modalité d'énonciation exclamative"46,"Mais...rien.!".Outre sa fonction émotive,cette modalité est un signal de fin de tour de nature paraverbale ${ }^{47}$ du fait de sa courbe intonative ascendante.Ce qui requiert une prise de position de la part de Paul. À la recherche du consensus avec sa femme, Paul lui répète son discours antérieur ${ }^{48}, " T u \ldots$...ptimistes", et le fait suivre d'une assertion à valeur de justification,"Antoine...le prévenir".Ne pouvant pas démentir son mari,Marine choisit de mettre un terme à leur échange.Le caractère clôturant du dernier tour est indiqué par "une chute de l'intensité articulatoire" ${ }^{\text {"49 }}$.Ce signal prosodique est dénoté par le discours attributif,"concéda-t-elle",qui 
précise également"(...)l'intensité avec laquelle elle(la parole) est prononcée" 50 , "d'une voix rauque".

Il apparaît donc que Marine et Paul se sont efficacement engagés à assurer le maintien du parcours alternatif des tours. Chaque interlocuteur enchaîne par une prise de parole qui répond aux sollicitations de son partenaire communicatif.Cette pratique collective $\mathrm{e}^{51}$ de la parole relève de la " "synchronisation interactionnelle" (ou intersynchronisation...)" ${ }^{52}$ qui "(...)consist(e) dans le fait qu'ils(les interlocuteurs )ajustent ,coordonnent, harmonisent en permanence leurs comportements respectifs $(. . .)^{153}$.

Cette intersynchronisation sous-tend les trois propriétés du système des tours:

"1-La fonction locutrice doit être occupée successivement par différents acteurs" ${ }^{\prime 54}$ :

Le déroulement de la parole entre $L_{1}$ (Marine ) et $\mathbf{L}_{2}$ (Paul) s'effectue sur la base du respect du pacte d'interlocution"qui stipule que les rôles doivent s'inverser et que chacun des interlocuteurs deviendra à son tour énonciateur(...)"

L'aspect configurationnel du schéma interlocutif du dilogue obéit donc au"principe d'équilibrage des tours" ${ }^{156}$ qui porte sur:

-Le nombre des tours:

"(...)dans les interactions duelles,ce nombre est nécessairement le même,à une unité près(lorsque c'est le même locuteur qui ouvre et clôt l'interaction) pour les deux participants." ${ }^{\text {57 }}$ :quatre tours sont assurés par Marine,responsable de l'ouverture/la clôture de l'échange ,et trois par Paul. 
-La focalisation du discours:

Ni Marine, ni Paul ne tiennent un discours "autocentré" ${ }^{58}$.Chaque locuteur tend à articuler son discours avec celui de son destinataire en vertu des signaux de fin de tour.

"2-Une seule personne parle à la fois"

Le dilogue est dépourvu de différents cas de chevauchement $^{60}$ qui accompagnent "les interruptions" ${ }^{\prime 61}$.

"3-Il y a toujours une personne qui parle,c'est-à-dire que le temps de la conversation est pour l'essentiel occupé par de la parole(...),et que les intervalles("gaps") séparant les tours sont eux-aussi réduits au minimum." ${ }^{62}$ :

Les points de suspension dans le premier tour de Marine et le deuxième tour de Paul marquent des "auto-interruptions"63 qui entraînent des"pauses,ou silences"intra-répliques"'"64.

Mais,ces pauses ne peuvent être considérées comme des "ratés" 65 ou des "phénomènes d'arythmie et d'asynchronie" 66 puisqu'elles n'ont pas"(...) des effets notables et généralement négatifs sur le déroulement de l'interaction." ${ }^{67}$. En effet,elles révèlent l'état d'âme des interlocuteurs.La première auto-interruption de Marine,"c'est l'heure...", trahit son hésitation à déclarer à Paul son intention d'aller au travail ,le lendemain de leur mariage, d'autant plus qu'elle a passé la nuit de noces ${ }^{68}$ aux urgences.

Quant à la deuxième auto-interruption,'il n'y aura rien de changé....",elle produit une transition entre le but de la conversation de Marine avec son mari et la question qu'elle lui pose. Ce qui contribue à donner plus de relief à cet acte interrogatif,déclencheur du dilogue des époux. 
Parallèlement à Marine,Paul se coupe la parole deux fois à la file,"Si Vincent ne...,mourir...".Il laisse ainsi voir un trouble profond face à sa femme"affolée",et à l'état critique de son frère.

Ces propriétés s'appliquent plus ou moins au polylogue des Soubeyrand:

"Bien avant l'heure habituelle,Emma avait poussé dehors les derniers clients.Une fois le rideau de fer baissé,elle rejoignit Marie-Angèle ${ }^{69}$,Antoine et Paul dans la cuisine,dont elle referma soigneusement les fenêtres.Seule Sophie,retenue au tribunal,manquait à la réunion de famille.Emma exposa la situation aux autres de manière assez brutale.

T1.1 ${ }^{70}{ }^{-1 M e s}$ lettres iront par deux ${ }^{\prime \prime 11}$,ce salaud nous annonçait donc son courrier aux gendarmes!Pour la partie:"Finis les jours heureux",je crois qu'on a du souci à se faire!

Antoine et Paul,toute rivalité oubliée,échangèrent un regard plein d'angoisse.

T1.2-Je ne comprends pas,conclut Emma en jetant la lettre anonyme sur la table devant ses fils.Cet immonde corbeau sait probablement tout,et depuis sept ans...Pourquoi a-t-il autant attendu?Mystère!

Il y eut un long silence puis Antoine suggéra, d'un ton rageur:

T2-Parce que je suis revenu?

Sa mère le regarda droit dans les yeux mais ne répondit pas,et ce fut Marie-Angèle qui prit la parole,très calmement.

T3-Et après?Quelqu'un a quelque chose à se reprocher,ici? La seule erreur a été de se taire,je ne vous apprends rien.Si vous vous décidez à parler avant que 
votre mystérieux correspondant le fasse à votre place,ce sera un moindre mal.

T4-Jamais!riposta Emma.

T5-Mais pourquoi,à la fin? C'est de la folie,vous allez tous finir en prison!

T6-Elle a raison,lâcha Antoine amèrement.De toute façon,ils vont rouvrir l'enquête,poser des questions...

T7-Surtout à toi,murmura Paul,tu étais son associé.

T8-C'est bien pour ça que tu dois repartir,ils n'iront pas t'interroger à São Paulo,dit Emma dans un souffle.

Elle s'accrochait à son idée,celle qu'elle avait défendue si longtemps et si âprement comme la seule solution possible. ${ }^{, 72}$

Le schéma participatif de cette réunion familiale est fixé d'entrée,"Bien ...famille".Il regroupe quatre participants ratifiés:Emma,Marie-Angèle,Antoine et Paul.C'est Emma qui engage le polylogue.L'indication de la locutrice de la première prise de parole passe par le discours attributif:"Emma exposa la situation aux autres de manière assez brutale ${ }^{173}$.

Le comportement gestuel accompli par Emma ,avant la fin de son tour, permet de déterminer ses successeurs potentiels:"en jetant la lettre anonyme sur la table devant ses fils". Ce procédé de sélection non verbal dénote"l'allocation partiellement libre(...)" ${ }^{174} \mathrm{du}$ tour dans la mesure où Marie-Angèle est exclue par la locutrice de la succession.

C'est Antoine qui se sent plus directement concerné que Paul par la question qui clôt le tour de sa mère $^{75}$, "Pourquoi...attendu?".En effet,"(...),il est très rare qu'une question en"pourquoi(ne pas)"fonctionne comme une simple demande d'explication:l'énoncé se charge très systématiquement d'une valeur 
seconde,mais souvent dominante de suggestion,critique,reproche ou protestation. ${ }^{176}$.

Antoine occupe le tour et choisit son allocutaire par le biais d'un procédé verbal:il répond à sa mère par une question qu'il lui adresse,"Parce que je suis revenu?".Mais, Emma s'abstient de prendre la parole.D'où "l'intrusion" ${ }^{77}$ de Marie-Angèle qui s'autosélectionne:"ce fut Marie-Angèle...calmement.". Cette intrusion"est d'autant plus incontestable que la nature du successeur est clairement déterminée(...)" ${ }^{178}$.Elle fait

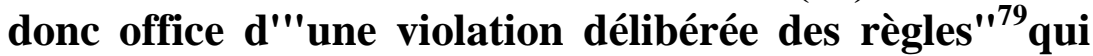
porte atteinte à l'homogéniété organisationnelle du schéma interlocutif:

"(...)il peut arriver que $\mathbf{L}_{3}$ (Marie-Angèle) réponde par "intrusion à une intervention initiative produite par $L_{(2)}$ à l'intention de $L_{(1)}$-l'échange pouvant alors être dit,du point de vue de son schéma interlocutif "hétérogène"." 80

Cette hétérogéniété est provoquée par le bouleversement des rôles interlocutifs:destinataire secondaire par rapport au "dyade"81,

EmmaAntoine,Marie-Angèle se transforme ,par son intrusion, en destinataire privilégiée.

Mais, la désorganisation du schéma interlocutif ne nie en rien l'importance de cette intrusion légitimée aussi bien par le statut ${ }^{82}$ de Marie-Angèle que par son effet positif sur la poursuite de l'échange verbal.En assumant la responsabilité interlocutive de sa partenaire communicative,Marie-Anègle empêche l'obstruction du canal discursif et garantit le maintien de la succession des tours. 
Ce qui mène à la réussite de l'intrusion au niveau de la relation interlocutive des participants ratifiés:

"Il y a réussite si $\mathbf{L}_{3}$ parvient finalement à s'imposer auprès comme son interlocuteur légal." ${ }^{83}$.

de

Emma se présente comme successeur de sa cadette qui s'adresse à l'ensemble des allocutaires par le déictique de personne ${ }^{84}$ "vous" ${ }^{85}$.

L'indication des locuteurs des tours suivants dont l'allocation est totalement libre s'opère à travers la technique de l'auto-sélection soulignée par:

-Le contenu des paroles qui appartiennent à une locutrice précise, MarieAngèle:"Mais....prison."

-Les différents verbes attributifs:"lâcha Antoine, murmura Paul, dit Emma."

Le relais de la parole entre ces différents locuteurs passe par plusieurs signaux de fin de tour.

Le premier tour de parole est interrompu par la narratrice :"Antoine et Paul...d'angoisse." Ce qui est dénoté,dans T1.2, par le verbe attributif "conclut $E m m a$ "dont la valeur déductive marque la clôture de la prise de parole de $L_{1}($ Emma).Cette dernière indique la fin de son tour par des signaux de nature verbale,une question,"Pourquoi...attendu?",et de nature prosodique,une exclamation,"Mystère!".Le caractère intiatif de l'acte interrogatif est accentué par l'intonation ascendante de la modalité exclamative.Antoine enchaine par une question rhétorique, "Parce que... revenu ?",qui"n'appelle pas de véritable réponse,mais tout au plus une réaction analogue à celle qu'entraînent habituellement les énoncés assertifs $(. . .)^{\prime 86}$. 
Marie-Angèle réagit au signal de son neveu.Elle remplit la place transitionnelle de son tour par un énoncé hypothétique,"si vous vous....mal",qui ,aux yeux d'Emma $^{87}$, prend la valeur d'une provocation:

"(...),il y a des types d'assertion qui incitent plus que d'autres à réagir,(...):les" provocations",attaques,ou contre-vérités éhontées,qui entraînent de la part de $L_{2}$ une protestation ou une réfutation(...)"

La protestation d'Emma se traduit par une mélodie exclamative montante indiquée par le verbe attributif "riposta".C'est par ce signal prosodique que MarieAngèle achève également sa prise de parole," $C^{\prime} e s t . . . e n$ prison!",qui correspond à une évaluation ${ }^{89}$ au sein de son échange avec sa soeur.

La récurrence de ces exclamations sert à conforter la tension qui conditionne le parcours fonctionnel des tours.

Antoine enchaîne.Mais,Paul l'empêche de terminer son tour en lui coupant la parole"Surtout...associé".Le caractère délibéré de cette hétéro-interruption tient au fait que:

" $L_{2}$ (Paul) s'empare de la parole alors que $\mathrm{L}_{1}$ (Antoine) continue à parler,et que rien ne permet de soupçonner l'apparition d'une place transitionnelle(aucun signal de fin de tour) (...).C'est un petit coup de force exercé par $L_{2}$ contre $L_{1}$, une forme de violation territoriale. ${ }^{90}$

En effet ,Paul sort de son mutisme non pour proposer une solution mais pour rappeler à son frère la menace qui lui pèse comme du plomb.Emma profite de l'aubaine.Elle intervient et déclare la fin du polylogue en réclamant à Antoine d'avoir recours à la seule échappatoire,repartir au Brésil ,à travers un 
signal verbal qu'est "la modalité d'énoncé"191 "déontique" "92:"tu dois repartir."

L'emplacement du changement du tour se superpose à la nature du successeur pour illustrer le schéma interlocutif en fonction des propriétés du système des tours.

Ce schéma est constitué de huit tours de parole répartis en: un trilogue entre $\operatorname{Emma}\left(L_{1}\right)$-Antoine $\left(L_{2}\right)$ - Marie-Angèle $\left(L_{3}\right)$ de $T 1.1, T 1.2$ à $T 3$ suivi d'un autre dilogue entre $\operatorname{Emma}\left(L_{1}\right)$-MarieAngèle $\left(L_{2}\right)$ de $T 4$ à $T 5$ qui s'achève en un trilogue entre $\operatorname{Antoine}\left(L_{1}\right)-\operatorname{Paul}\left(L_{2}\right)-\operatorname{Emma}\left(L_{3}\right)$ de $T 6$ à $T 8$.

Il devient donc évident qu'Emma prend la parole pour la plupart du temps dans le polylogue:

"Le principe d'équilibrage des tours est alors sérieusement mis à mal,en ce qui concerne non seulement leur longueur,mais aussi le nombre des tours assurés par les différents participants." ${ }^{193}$.

Ce qui ne signifie pas "la monogestion" ${ }^{94}$ de l'échange discursif: il y a "(...)une différence fondamentale entre conduire une interaction et la gérer seul.Toute interaction implique en effet une co-action de sorte que si l'un veut conduire encore fautil que l'autre accepte d'être conduit et participe ainsi à la gestion de l'échange." ${ }^{95}$.

Grâce à son statut ${ }^{96}$, Emma est convoquée à la place "de gestionnaire de l'échange" ${ }^{17}$ par ses partenaires communicatifs qui oeuvrent à synchroniser leur attitude interlocutive en réagissant à ses prises de parole. 
Or, la succession de la parole au cours du déroulement du polylogue est entravée par:

-Le chevauchement ${ }^{98}$ qui accompagne l'hétérointerruption délibérée de Paul.Ce qui produit un effet de "carambolage verbal"99 dans l'enchaînement alternatif des tours.

-La pause inter-réplique ${ }^{100}$ qui fragmente le premier tour de parole d'Emma en deux parties.La narratrice intervient par un commentaire pour indiquer l'effet de la parole d'Emma sur ses fils ${ }^{101}$ qui éprouvent un cuisant sentiment d'angoisse face à la situation dans laquelle ils s'empêtrent.

-La pause intra-réplique produite par l'absence de réponse d'Emma au tour de parole d'Antoine.Il s'agit d'une "semi-troncation ${ }^{102}$ ( dans la mesure où l'absence de production verbale est ici "marquée")"103 par le regard de reproche d'Emma qui ne veut effectivement pas que la conversation tourne à un affrontement qui la dresse contre son fils:

"(...) au fond d'elle-même elle faisait confiance à Antoine,certaine qu'il ramènerait toujours les trois autres à bon port.Ce fils-là était son premier enfant,peut-être son préféré $(. . .)^{\prime \prime 104}$.

Ces pauses révèlent donc la perturbation de l'état d'âme des locuteurs qui se traduit par le dysfonctionnement de leurs prises de parole.

C'est également l'état d'âme d'Antoine qui bloque son parcours interlocutif lors de l'interrogatoire du juge Herrero $^{105}$ :

" T1-Nous y voilà! Claironna Herrero en désignant les résultats du laboratoire de la police.C'est ce que j'espérais depuis le début,mon intuition ne m'avait pas trompé,maître... 
D'un geste triomphant, il poussa les feuillets dans la direction de Claire.Elle parcourut le rapport en quelques instants,pâlissant au fur et à mesure de sa lecture.Quand elle se tourna enfin vers Antoine,il vit qu'elle en restait sans voix.

T2-Antoine Soubeyrand,c'est bien dans votre véhicule que le corps de Laurent Labaume a séjourné,martela le juge.Si vous voulez demander une contreexpertise,ne vous gênez pas!

Après un bref silence,Antoine se contenta d'ébaucher un geste d'impuissance. Nier l'évidence ne lui servirait à rien,puisque la preuve était établie.

T3-Vous avez une explication à me fournir ?insista Herrero.

Un nouveau silence,menaçant,contraignit Claire à murmurer.

T4-Antoine ?Souhaitez-vous vous entretenir avec moi?

T5-Vous aurez tout loisir de parler à votre client,maître,mais pour l'instant c'est moi qui aimerais l'entendre! À toutes fins utiles,j'ai procédé à une vérification:il n'y a pas trace d'une plainte pour vol de voiture ,à l'époque des faits.Ce $4 x 4$ était le vôtre,Antoine Soubeyrand,et vous y avez transporté un cadavre!

S'en souvenir rendait Antoine malade et il serra les dents.De toute façon,il ne regrettait rien,sauf peut-être d'avoir écouté sa mère.

T6-Très bien,tranche Herrero,je vous inculpe donc du meurtre de Laurent Labaume,perpétré la nuit du 14 juillet 1996. Vous allez regagner votre cellule jusqu'au procès .

Apparemment très content de lui,il fit signe à son greffier d'appeler le gendarme qui attendait dehors.Résigné, Antoine ne réagit pas quand on lui remit les menottes.Qu'aurait-il pu faire d'autre, à présent? 
T7-Antoine,écoutez-moi!lui jeta Claire d'un ton pressant.Je viendrai à la prison cet après-midi,il faut que vous me parliez.Je ne peux pas vous défendre si vous ne me dites pas la vérité!

Il se laissa entraîner par le gendarme mais, au bout du couloir,il regarda par-dessus son épaule.Claire se tenait très droite, frêle dans sa robe d'avocate,petite bonne femme obstinée qu'il commençait à connaître et à respecter.Elle était son seul vrai lien avec l'extérieur.Devait-il se confier à elle?" 106

À l'opposé du dilogue et du polylogue précédents,il s'agit ici d'une situation communicative formelle basée sur un rapport inégalitaire qui"(...)fait apparaître une position "haute" corrélée à une position"basse" "107 où "l'on se trouve l'obligé d'un partenaire qui a la responsabilité de l'échange et qui dispose de savoirs ou de pouvoirs consacrant sa "supériorité" "108.

Alors que la position haute est occupée par le juge Herrero,la basse l'est par Antoine et son avocate,Claire.Cette organisation hiérarchique du schéma participatif "ne saurait être vexatoire.Le caractère institutionnel de ce rapport de places(relation) et l'aspect nettement spécialisé de ces interactions conduisent les sujets à accepter cette dissymétrie constitutive." 109

Ainsi Herrero prend-il l'initiative de la parole.Il est indiqué par le verbe attributif "claironna".II sélectionne son allocutaire par un indice d'allocution de nature verbale,l'appellatif ${ }^{10}$ "maître" qui désigne Claire.Cette dernière a recours à un procédé de sélection non verbal,l'orientation du corps:"elle se tourna enfin vers Antoine".Mais, Antoine refuse de faire figure de successeur. Herrero s'auto-sélectionne et s'adresse à son destinataire à travers des procédés verbaux:la nomination explicite"Antoine Soubeyrand" suivie d'une 
reprise anaphorique ${ }^{111}$ pronominale"vous ${ }^{\prime 112}$. Antoine se mure dans son silence.

Toutefois,le refus de l'accusé d'accomplir son devoir de succession n'affecte pas le caractère opérationnel du trilogue en ce sens que:

"Tous les destinataires d'un message,même ceux qui ne sont de toute évidence qu'indirects(...)jouent un rôle important dans le déroulement de l'interaction. "113

Le rôle d'Antoine est d'une importance capitale d'autant plus qu'il est sélectionné comme destinataire privilégié par ses partenaires communicatifs.Plus précisément,le silence de l'accusé ne met pas un terme à l'interrogatoire mais devient un catalyseur qui entraîne des intrusions successives de la part:

-du juge Herrero:qui essaie"de sauver la mise en reprenant la parole" ${ }^{\prime 114}$ dans le $3^{\text {ème }}$,le $5^{\text {ème }}$ et le $6^{\text {ème }}$ tours de parole à la place du successeur déterminé par"Antoine" et le déictique "vous".

-de Claire que le silence"contraignit" de se substituer à son client , désigné par "vous", dans la 4ème et la 7ème prises de parole.

Loin de constituer "(...)des tentatives de parasitage de la relation interlocutive" ${ }^{\prime 115}$ à cause de l'hétérogéniété du schéma interlocutif qui en résulte,ces intrusions ont donc contribué pour une grande part à assurer cette relation.En effet,ni le juge,ni l'avocate ne se sont obstinés à remettre l'accusé à sa place de destinataire privilégié et ,par la suite, à interrompre l'enchaînement successif de l'interrogatoire.Au contraire,ils se sont engagés activement dans l'échange discursif conformément au devoir interlocutif inhérent à leur statut: en raison de l'autorité que lui confère son pouvoir,Herrero représente le gestionnaire officiel de l'échange"(...)lequel doit assumer,(...),la responsabilité 
de sa réussite ou de son échec(...)"116. Quant à Claire,elle est tenue d'intervenir autant que possible pour prendre la défense de son client.

Le respect de ce devoir garantit également le fonctionnement du changement de tour entre les interlocuteurs.

Le juge achève la première prise de parole par un signal de nature mimo-gestuelle:"D'un geste triomphant, il poussa les feuillets dans la direction de Claire".Cet "ANL"117 "joue un rôle complémentaire"118 par rapport à l'énoncé de Herrero :

"(...)en situation de face à face,les regards et les gestes contribuent largement , aux côtés des marqueurs linguistiques,à la gestion des tours de parole et au bon fonctionnement de la "synchronisation interactionnelle"'"119

Par son geste,le juge met entre les mains de l'avocate la preuve irréfutable de l'accusation d'Antoine et requiert,de ce fait, une réaction de sa part. Claire répond par un ANL qui "assum(e) un rôle plein"120 en ce sens que "Le comportement non verbal assure à lui seul la réalisation d'une intervention initiative(...) ou réactive(...)le comportement non verbal fonctionne exactement comme un acte de langage"121:"Elle parcourut le rapport en quelques instants".Cet ANL reflète l'état d'âme de l'avocate "pâlissant au fur et à mesure de sa lecture.". Claire est tellement saisie qu'elle se trouve privée de toutes tentatives de défense.Elle signale la fin de son tour en se dirigeant vers son client dans l'espoir d'obtenir une nouvelle information lui permettant de plaider en sa faveur.Mais, Antoine emboîte le pas à son avocate qui "en restait sans voix".Il est sollicité par le juge qui termine son tour en lui déclarant:"Si vous voulez...pas!".Les exclamations ,qui ponctuent la fin 
des prises de parole de Herrero, prennent la valeur illocutoire d'un acte interrogatif ${ }^{122}$ en vertu du "cadre interactif" ${ }^{\prime 23}$ de l'interrogatoire dont le critère fonctionnel repose sur l'utilisation d'un questionnaire suivi par l'interrogateur.

Antoine se contente d'accomplir "un geste d'impuissance".Face à cette semi-troncation, le juge tend à "déclencher chez le questionné un mécanisme associatif quelconque (...)" ${ }^{124}$ en lui posant une autre question : "Vous avez...fournir?".

En dépit du caractère contraignant ${ }^{125}$ des signaux de fin de tour auxquels il a eu recours, Herrero ne parvient pas à briser le silence de l'accusé.Ce qui incite Claire à intervenir pour appeler une réaction de la part de son client à travers une double interrogation:"Antoine?souhaitez-vous...moi?".

Cependant,Antoine continue à faire la sourde oreille et à la question de l'avocate et à l'exclamation suivante du juge:"Ce $4 x 4$....un cadavre!".Il ne réagit à ces signaux que par une simple mimique:"il serra les dents.".Herrero intervient donc pour clore l'interrogatoire:"(...),il fit signe à son greffier d'appeler le gendarme"qui remet les menottes à l'accusé et le conduit à sa cellule. Claire a beau solliciter son client en se servant d'un dernier signal prosodique qu'est le "ton pressant" de son énoncé exclamatif:"Je ne peux pas...la vérité!".Sans un mot,celui-ci"se laissa entraîner par le gendarme.".

Cette absence de toute participation interlocutive d'Antoine se répercute sur les propriétés du système des tours.

Le schéma interlocutif de l'interrogatoire ne s'organise pas suivant le principe d'équilibrage des tours.La parole se déroule effectivement entre l'avocate et le juge qui a le dessus tant sur le niveau du nombre 
des tours que sur celui de leur longueur:cinq tours sont remplis par Herrero contre deux par Claire. Cette dernière n'ose pas interrompre le discours de son supérieur. Ce qui explique l'absence de toutes formes de chevauchement. Quant au silence,il acquiert deux différentes formes:

-La pause intra-réplique produite par le juge qui se coupe la parole dans sa première prise de tour.Cette auto-interruption soulignée par un renvoi à la ligne marque le passage du verbal,"Nous $y$ voilà...maître...",au non verbal qui en constitue la matérialisation directe,"D'un geste...Claire."

-Les pauses inter-répliques qui se réalisent sur deux modes:

-implicite du "fait que l'absence de réaction soit(...)camouflée"126 : à la suite de l'ANL de Claire,"Elle parcourut...Antoine",rien n'est dit sur le silence d'Antoine qui"vit...voix".

- explicite dans la mesure où la narratrice choisit:

- soit de "poursuivre le discours des autres en soulignant l'absence de réponse"127:"Un nouveau silence ,menaçant ,contraignit Claire à murmurer" : non seulement la narratrice décrit le silence par "menaçant", mais encore met en relief son effet contraignant sur l'avocate.

-soit de" se concentrer sur le mutisme de celui qui se tait" ${ }^{128}$ pour décrire ses gestes ou sa mimique.Ce qui apparaît à trois reprises:

-"Après un bref silence...établie":face au bienfondé de son accusation,Antoine se sent tellement désarmé qu'il ne tarde pas à exprimer son impuissance par un geste suivi d'un commentaire à fonction justificatrice, "Nier l'évidence....établie." 
-"S'en souvenir....mère" souvenirs,l'accusé esquisse une mimique,"serra les dents",qui laisse voir son incapacité de contester les paroles du juge ${ }^{129}$. Le commentaire qui s'ensuit,"De toute façon...mère", reflète l'état d'âme d'Antoine qui réalise que le regret n'a pas de valeur puisque la partie est perdue.

-"Résigné...à présent?" :l'accusé obtempère donc à l'ordre du juge.

Il appert que le silence joue un rôle crucial dans le déroulement discursif de l'interrogatoire.Catalyseur d'intrusions ${ }^{130}$,il devient également révélateur de l'état d'âme qui conditionne l'attitude interlocutive d'Antoine et détermine,par conséquent, la réaction des autres interlocuteurs à son égard.Pour Herrero, le silence persistant d'Antoine ${ }^{131}$ correspond à une impuissance de se défendre qui implique un aveu d'auto-accusation .Ce qui mène le juge à l'inculper du meurtre de Labaume.Mais,pour Claire,par ce silence,son client cache une vérité condamnable qui l'accule à une position de faiblesse.Ce qui la rend incapable de le défendre "Je ne peux pas...la vérité!".

Le silence gagne ainsi le statut illocutoire d'un ANL qui joue un rôle plein et s'agence,par le fait même,avec les tours de parole en vertu de l'intersynchronisation.

Tout au long de notre étude,nous nous sommes efforcée de dégager les règles qui régissent le fonctionnement du système des tours.La mise en oeuvre de ces règles est soulignée par le parcours discursif du trilogue,du polylogue et du dilogue des Soubeyrand.

À chaque type de ces interactions verbales, correspond un schéma participatif dont l'aspect configurationnel diffère tant sur le plan structural que sur le plan opérationnel.En dépit de cette 
différence,tous les participants ratifiés sont tenus de respecter le principe d'alternance pour assurer la succession des tours de parole.À cette fin,ils ont recours à plusieurs procédés verbaux/non verbaux susceptibles de déterminer la nature du successeur.Ce dernier marque l'emplacement du changement de tour à travers des signaux de fin de tour de nature verbale/non verbale,paraverbale.

Or ,cette collaboration se trouve entravée par certains interlocuteurs qui ,pour une raison ou une autre, choisissent de ne pas synchroniser leur attitude interlocutive par la réponse aux sollicitations de leurs partenaires communicatifs.Ce qui entraîne les ratés des tours: les hétéro/autointerruptions,les intrusions,et le silence ou les pauses inter/intra-répliques.Autant ces ratés interrompent l'enchaînement des tours,autant ils contribuent à maintenir cet enchaînement et à clarifier la situation communicative en trahissant le trouble de l'état d'âme des interlocuteurs.

Cette organisation fonctionnelle des tours est illustrée par l'analyse des propriétés qui montre jusqu'à quel degré les participants ratifiés sont parvenus à s'adapter aux règles du système.

Bref,nous espérons avoir jeté des fondements théoriques et exposé des principes sur lesquels doivent se concevoir des investigations du champ des tours qui laissent aux chercheurs leur part de création renouvelée. 


\section{Notes}

1)Françoise Bourdin est"née (en 1952) à Paris de parents chanteurs lyriques"

http://www.babelio.com/auteur/Francoise-Bourdin/15504 consulté le 18/05/2014.

2)BOURDIN(F.), Les Soleils mouillés, Paris, Éd. Julliard, 1972.

3)ID, Terre indigo, Paris, Éd.Belfond, 1996.

Plusieurs romans de Bourdin ont été adaptés à la télévision.

4)http://www.pocket.fr/site/francoise_bourdin_\&181\&5827.html consulté le 17/06/2014.

5)http://bibliobs.nouvelobs.com/romans/20120525.OBS6599/franc oise-bourdin-l-ecrivain-qui-vend-500-000-livres-par-an-sans-fairede-bruit.html consulté le 20/05/2014.

Il convient de souligner que BOURDIN(F.) n'a pas été couronnée par de prix littéraires en raison de l'absence de couverture médiatique de son oeuvre:

"Pourquoi un tel silence? Une chose est sûre, Françoise Bourdin n'appartient pas au sérail germanopratin. On ne la verra pas dans un cocktail mondain, elle a bien essayé mais elle s'y ennuie profondément, souffre d'entendre des auteurs dans le vent se "gargariser à longueur de soirées de vendre beaucoup plus de livres que dans la réalité". http://www.lejdd.fr/Culture/Livres/Actualite/Francoise-Bourdin-linconnue-des-best-sellers-491514 consulté le 7/06/2014.

6)BOURDIN(F.), Un été de canicule, Paris, Éd.Belfond,2003.

7)La conversation représente l'"exemple type d'interaction (verbale) informelle".

VION(R.), La communication verbale.Analyse des interactions, Paris,Éd.Hachette,1992,p.128.

8)Antoine s'est associé avec Cantel et Labaume pour ouvrir une discothèque. Ce dernier est disparu la nuit du 14 juillet 1996.Cette disparition subite n'a pas été expliquée.Mais, Antoine était considéré comme le principal suspect qui pourrait avoir pour mobile le désir de se venger de Labaume qui lui avait fait faire six mois de prison pour trafic de drogue.Antoine a donc été obligé de partir pour ne pas être arrêté par la police. 
9)Les règles d'analyse des interactions verbales "opèrent à des niveaux différents:

(1)Règles qui permettent la gestion de l'alternance des prises de parole,c'est-à-dire la construction de ces unités formelles que sont les "tours".

(2)Règles qui régissent l'organisation structurale des interactions verbales,et qui permettent la mise en séquence de ces unités fonctionnelles que sont les échanges et les interventions.

(3)Règles enfin qui déterminent la construction de la relation interpersonnelle -sur un mode intime ou distant,égalitaire ou hiérarchique,consensuel ou conflictuel-"

KERBRAT-ORECCHIONI(C.), Les interactions verbales.Approche interactionnelle et structure des conversations, T.I,Paris,Éd.Armand Colin/Masson,1998(Troisième édition) ,p.157.

Cet ouvrage va constituer le point de repère de notre étude qui portera sur l'analyse des règles du premier niveau à travers la mise en parallèle de trois différentes formes d'interactions verbales.

10)ibid,p.159.

11)Loc.Cit.

12)Ce système est résumé par "la formule ababab" ibid,p.160.

Cette formule illustre l'engagement mutuel des locuteurs dans le processus communicatif : à chaque $L 1$ doit succéder $L 2$.

13)KERBRAT-ORECCHIONI(C.) explique l'utilisation de la terminologie anglaise en soulignant que:

"la terminologie étant,du fait de son ancienneté relative,mieux fixée en anglais qu'en français,s'il arrive que le terme d'origine ait en français un équivalent fort acceptable(...),il arrive aussi malheureusement qu'aucune traduction ne satisfasse à la double condition de clarté et d'économie(...).D'où la nécessité dès que l'on aborde ces matières de pratiquer un "code switching" permanent(en voici du reste un premier échantillon -en attendant que se lexicalise au même degré l'expression d'alternance codique")"ibid,p.6.

14)ibid,p.160.

15)ibid,p.165. 
16)Antoine et Marine "s'étaient aimés passionnément, et un beau matin il avait filé sans prévenir ,la quittait comme si elle n'avait aucune importance.(...). Il n'y avait pas eu de dispute entre eux,pas de lettre pour justifier cette soudaine rupture.Du jour au lendemain,il avait disparu au bout du monde."

BOURDIN(F.),Op.Cit,p34.

Il est à noter que Marine"travaillait en tant qu'infirmière"ibid,p13,dans l'hôpital d'Apt où Vincent a été transporté après son accident.

17)"T1"désigne tour de parole numéro1,etc.

18)BOURDIN(F.),Op.Cit, pp.18-19.

19)Les composantes de base de la situation communicative des interactions verbales sont:le cadre spatio-temporel, le but et les participants.

Voir KERBRAT-ORECCHIONI(C.), Les interactions verbales.Approche interactionnelle et structure des conversations,T.I,pp.75-111.

20)"(...),deux types de dialogue sont à distinguer:

-Le dilogue,c'est-à-dire le dialogue à deux personnages qui,tout en étant reliés par différents axes relationnels,poursuivent chacun un objectif propre qui les expose éventuellement à entrer en conflit;

-Le polylogue qui fait intervenir plusieurs personnages,et pose par suite des problèmes spécifiques à l'auteur:problèmes de locuteur,de point de vue,d'enjeu,de stratégie(...)"

BERTHELOT(F.),Parole et dialogue dans le roman,Paris,Éd. Nathan Université, 2001,p.5.

Cette distinction est soutenue par KERBRAT-ORECCHIONI(C.) pour qui la définition traditionnelle du dialogue comme une parole échangée entre $L_{1}$ et $L_{2}$ est"(...) surprenante, d'un point de vue étymologique d'abord,car le mot est formé à l'aide du préfixe"dia"("dialogue"= parole"qui traverse",qui circule et s'échange), et non de "di-":il s'agit là d'un fait de remotivation étymologique,comme il s'en produit souvent dans les langues et qui n'a en soi rien de scandaleux -tant qu'on ne se réfère pas malencontreusement à l'étymologie supposée du terme pour justifier un usage étroit,ou qu'on ne s'excuse pas auprès de cette étymologie d'une utilisation plus large(...)"

KERBRAT-ORECCHIONI(C.), Les interactions verbales .Approche interactionnelle et structure des conversations,T.I,p.116. 
21)ibid,pp.84-85.

Le schéma participatif " se décompose en un "format de production"et un"format de réception".(...).Le format de production est rempli par le locuteur,personne unique(...) et aisément identifiable comme source de production des signifiants(...)"ibid,p.85.

Quant au format de réception,il regroupe les participants ratifiés et les spectateurs:

"Les participants ratifiés font officiellement partie du groupe conversationnel,ainsi qu'en témoignent surtout "l'arrangement physique" de ce groupe, et le comportement non verbal de ses membres(...): le message produit par l'émetteur les concerne à des degrés divers,alors que les"bystanders" ne sont que les témoins ou les spectateurs d'un échange dont ils sont en principe exclus." ibid,p.86.

Chaque locuteur est tenu de remplir alternativement les deux formats.

22)Un destinataire peut être "(...) de façon secondaire,par rapport au(x) destinataire(s) privilégié(s) à qui s'adresse surtout le parleur." ibid,p.90.

Le destinataire secondaire n'apparaît que dans un schéma participatif formé de plus de deux participants ratifiés.

23)ibid,p.169.

24)Il y a "(...) deux techniques d'allocation du tour, et de sélection du "successeur":

1-C'est $L_{1}$ qui sélectionne $L_{2}(\ldots)$,à l'aide de certains procédés verbaux et/ou non verbaux

1.Procédés verbaux:

.Nomination explicite de $\mathrm{L}_{2}(\ldots)$.

.Plus subtilement, et sur le mode implicite, le contenu des propos de $L_{1}$ peut jouer un certain rôle dans la sélection de $L_{2}$ :s'ils"concernent" plus directement $x$ que y,ou s'ils relèvent davantage de son domaine de compétence, $x$ apparaîtra comme un successeur plus légitime que $\mathbf{y}(. .$.$) .$ 
2.Plus importants sont les procédés non verbaux:geste d'ostension parfois,et surtout l'orientation du corps,et direction du $\operatorname{regard}(. .$.$) .$

2-Auto-sélection: $\mathrm{L}_{2}$ se sélectionne lui-même comme "successeur" de $L_{1}$." ibid,pp.169-171.

25)ibid,p.170.

26)BERTHELOT(F.),Op.Cit, p.168.

27)"(...) le narrateur se trouve tôt ou tard amené à interrompre l'échange verbal par un commentaire,une description,un élément de narration,pour le reprendre ensuite.Parfois,cette coupure a pour seule fonction de varier l'éclairage donné au lecteur sur la situation qui lui est rapportée.Parfois aussi,elle indique un silence entre les interlocuteurs.Enfin,elle correspond souvent à une ellipse du dialogue,une partie en étant alors résumée,voire escamotée." ibid, p.148.

28)Le locuteur"constitue l'instance qui adresse une parole à un allocutaire,l'allocutaire est l'instance qui se voit adresser cette parole. Le terme d'interlocuteur est un terme générique qui incarne le locuteur et l'allocutaire."

DURRER(S.),Le dialogue dans le roman,Paris,Éd.Nathan Université,1999,p.43.

29)KERBRAT-ORECCHIONI(C.),Les

interactions

verbales.Approche interactionnelle et structure des conversations,T.I,p.145.

30)BERTHELOT(F.), Op.Cit, p.183.

31) "(...) le changement de tour doit normalement s'effectuer à un point de transition possible(...).Cette place transitionnelle, $L_{1}$ doit l'inscrire d'une certaine manière dans l'énoncé à l'aide de l'un et/ou l'autre des signaux de fin de tour que permet le système."

KERBRAT-ORECCHIONI(C.),Les interactions verbales.Approche interactionnelle et structure des conversations,T.I,p.165.

32)Les signaux de fin de tour peuvent être de nature verbale,phonétique ou prosodique ,et mimo-gestuelle:

"(1) signaux de nature verbale:

.Complétude syntactico-sémantique de l'énoncé(...). 
.Statut illocutoire de l'énoncé:certains types d'actes(ex:les questions) ont plus que d'autres vocation à fonctionner comme la première partie d'une paire adjacente,donc à solliciter un enchaînement immédiat (...).

.Morphèmes connotant la clôture(" bon" , " voilà"...)" Loc.Cit.

33)Il serait pertinent de mettre en relief le trait distinctif des tours de parole par rapport aux actes de langage et des interventions:

" À la différence des actes et interventions,les tours sont pourtant en eux-mêmes dépourvus de toute valeur pragmatique(ce sont des unités purement formelles). "

KERBRAT-ORECCHIONI(C.),Les actes de langage dans le discours.Théorie et fonctionnement,Paris,Éd.Nathan,2001,p.62.

34)L'acte de langage"(...) désigne en tout état de cause tout acte réalisé au moyen du langage. " ibid, p.2.

Ceci s'explique par le fait que "dire,c'est sans doute transmettre à autrui certaines informations sur l'objet dont on parle,mais c'est aussi faire, c'est-à-dire tenter d'agir sur son interlocuteur,voire sur le monde environnant.Au lieu d'opposer comme on le fait souvent la parole et l'action,il convient de considérer que la parole elle-même est une forme d'action. " ibid,p.1.

35)ibid,p.92.

36) "L'élément Janus"renvoie à la bivalence du tour de parole dont" les deux interventions sont amalgamées au niveau de leur support signifiant" KERBRAT-ORECCHIONI(C.),Les interactions verbales.Approche interactionnelle et structure des conversations,T.I,p.241.

Ce qui se distingue du cas" où les deux interventions constitutives du tour sont réalisées par des signifiants distincts et successifs(...)."Loc.Cit.

37)"On voit que l'intervention ne se confond pas avec le tour de parole:unité fonctionnelle,elle ne se définit que par rapport à l'échange,et plus précisément,comme la contribution d'un locuteur particulier à un échange particulier. "ibid,p.225.

38)Il y a des interventions initiatives/réactives:

"(...)les premières donnent des droits ou imposent des contraintes à l'interlocuteur(...) tandis que les seconds sont positives ou négatives selon qu'elles satisfont ou non la fonction illocutoire de 
l'intervention

précédente."

BAYLON(C.),MIGNOT(X.),La communication,Paris,Éd.Nathan,1 997, p.198.

Il est à souligner que l'intervention initiative et celle réactive sont à la base de la paire adjacente"constituée de deux tours de parole en position de succession immédiate,prononcés par deux locuteurs différents(...)."

BANGE(P.),Analyse conversationnelle et théorie de l'action,Paris,Éd.Didier,1992,p.40.

39)"Toute conversation,et plus généralement toute forme de discours dialogué(...) se présente comme une architecture complexe et hiérarchisée fabriquée à partir d'unités emboîtées les unes dans les autres selon certaines règles d'organisation:

-Les actes de langage se combinent pour former des interventions,

-Lesquelles se combinent pour former des échanges,

-Lesquels se combinent pour former des séquences et en dernière instance,des conversations. "

KERBRAT-ORECCHIONI(C.),Les actes de langage dans le discours.Théorie et fonctionnement, p.61.

40)Ici,"La question est directe (ou explicite) d'un point de vue pragmatique,mais indirecte d'un point de vue grammatical. " ibid p.88.

Ceci est dû à l'absence du marqueur typographique, le point d'interrogation.Pour les autres marqueurs de la question Voir Loc.Cit.

41)On"(...) oppose les auto-reprises (de son propre discours) aux reprises diaphoniques (du discours de l'interlocuteur)."

VION(R.), Op.Cit,p.215.

42)Les reprises réitératives se distinguent"des reprises codées dans lesquelles l'acte de reprise est explicité par des formes linguistiques du type(quand je dis...ou quand vous dites...)." Loc.Cit.

43)Les reprises hétéro-déclenchées ont lieu"Lorsque l'énonciateur est amené à reprendre son propos suite à un comportement verbal ou non verbal de son partenaire(...)"ibid,p.217. 
44)"(...),certaines reprises ont une fonction idéelle dominante dans la mesure où elles visent notamment à s'assurer qu'on a bien compris." Loc.Cit.

45) KERBRAT-ORECCHIONI(C.),Les actes de langage dans le discours. Théorie et fonctionnement, p.64.

46)"Les modalités d'énonciation expriment l'attitude du locuteur par rapport à son allocutaire et se traduisent dans les types de phrase

suivants:assertif,interrogatif,jussif,exclamatif,négatif(...).L'excla mation est généralement classée à part,dans la mesure où elle permet d'exprimer les réactions subjectives et affectives du locuteur;elle relève de la fonction émotive."

CALAS(F.),CHARBONNEAU(D.-R.),Méthode du commentaire stylistique,Paris ,Éd.Nathan,2000,p.48.

47)Le matériel paraverbal est constitué des:"intonations,pauses,intensité articulatoire , débit , particularités de la prononciation, différentes caractéristiques de la voix."

KERBRAT-ORECCHIONI(C.),Les interactions verbales.Approche interactionnelle et structure des conversations,T.I,p.137.

48)À Marine qui demande:

"-Je peux aller le(Vincent) voir en salle de réveil?"

BOURDIN(F.),Op.Cit, p.14.

Le chirurgien répond:

"-Eh bien... À vrai dire,je ne suis pas franchement optimiste.Il est toujours dans le coma.La colonne vertébrale est touchée,je me suis fait assister d'un neurochirurgien." Loc.Cit.

49)KERBRAT-ORECCHIONI(C.),Les interactions verbales.Approche interactionnelle et structure des conversations,T.I,p.166.

50)BERTHELOT(F.),Op.Cit, p.166.

51)"(...)l'exercice de la parole loin d'être simplement une forme d"'expression de la pensée",est une pratique collective,où les différents participants mettent en oeuvre un ensemble de procédés leur permettant d'assurer conjointement la gestion du discours produit." 
KERBRAT-ORECCHIONI(C.),Les interactions verbales.Approche interactionnelle et structure des conversations, T.I,p.166.

52)ibid,p.20.

53)Loc.Cit.

54)ibid,p.160.

55)PERRET(M.), l'énonciation en grammaire du texte,Paris,Éd.Nathan,1994,p.11.

56)KERBRAT-ORECCHIONI(C.),Les

interactions

verbales.Approche interactionnelle et structure des conversations, T.I,p.160.

57) ibid,p.169.

58) ibid,p.161.

59) Loc.Cit.

60)Pour ces cas,Voir ibid,pp.174-175.

61)Il y a une interruption:"Chaque fois qu'un $L_{2}$ prend la parole alors que $L_{1}$ n'a pas fini son tour,on dira que $L_{2}$ "interrompt" $L_{1}$." ibid,p.173.

62) ibid,p.162.

63)Contrairement aux hétéro-interruptions où $L_{2}$ coupe la parole à $L_{1}$, les auto-interruptions renvoient aux"(...) inachèvements syntactico-sémantiques suivis d'une pause,dont est responsable lui-même le locuteur en place." ibid,p.173.

64)Ces pauses"apparaissent à l'intérieur d'un tour, et sont en quelque sorte la propriété exclusive du parleur." ibid,p.162.

65)Les ratés sont"les différents types de dysfonctionnements" qui affectent le système des tours . ibid,p.172.

Ces ratés se situent à deux niveaux:

."En ce qui concerne le moment de l'alternance" Loc.Cit.comme le silence et l'interruption.

.En ce qui concerne la nature du successeur"ibid,p.180.comme l'intrusion.

66) ibid,p.24.

67) ibid,p.157. 
68)C'est à cette nuit que l'accident de Vincent a eu lieu.

69)Marie-Angèle est la cadette d'Emma:

"À chaque coup dur,Marie-Angèle aidait Emma de son mieux.Elle l'avait tenue à bout de bras après la mort de son mari,s'était occupée activement de ses neveux et nièce auxquels elle était très attachée.Elle partageait tous les secrets de la famille,tous ses drames(...)"

BOURDIN(F.),Op.Cit, p.15.

70) $\mathrm{T1} 1$ renvoie à la première partie de $\mathrm{T} 1$ suivie de la deuxième partie dans T1.2.

71) Depuis le départ d'Antoine pour le Brésil,Emma recevait des lettres où il y avait"(...) des menaces à mots couverts et pas de signature." dit-elle à Marie -Angèle.

BOURDIN(F.),Op.Cit,p.17.

Après le retour d'Antoine,l'auteur de ces lettres anonymes a écrit à la gendarmerie pour désigner le puits qui contenait la dépouille de Labaume.

On va rouvrir l'instruction sur le meurtre de ce dernier dont l'affaire a été classée, sept ans plus tôt, faute de preuve.La situation devient donc pour Emma de plus en plus intenable.

72) ibid,pp.58-59.

73)On adjoint aux verbes attributifs:"(...) le groupe de ceux qui indiquent un sentiment ou une manière de parler."

BERTHELOT(F.),Op.Cit, p.166.

74)KERBRAT-ORECCHIONI(C.),Les verbales.Approche interactionnelle et structure des conversations, T.I,p.170.

75)Antoine a décidé de ne pas repartir au Brésil.Ce qui enrage Emma qui voit que la présence de son fils"(...)va nous attirer tous les ennuis de la terre"déclare-t-elle à Marie-Angèle.

BOURDIN(F.),Op.Cit, p.48.

Ainsi cherche-t-elle à le dissuader de rester à Cucuron:"Tout s'arrangera si tu t'en vas."'Lui dit-elle. ibid,p.91. 
76)KERBRAT-ORECCHIONI(C.),Les actes de langage dans le discours. Théorie et fonctionnement, p.96.

77)"Il y a différents types d'intrusions:

1- $L_{1}$ sélectionne $L_{2}$,mais c'est $L_{3}$ qui prend la parole en s'auto-sélectionnant alors que l'allocation du tour n'est en principe pas libre.

2- $\mathrm{L}_{1}$ ne sélectionne personne en particulier,mais c'est un "exclu", un interdit de parole,qui s'en empare(...)".

KERBRAT-ORECCHIONI(C.),Les interactions verbales.Approche interactionnelle et structure des conversations, T.I,p.180.

Ici ,il s'agit du premier type d'intrusion .Mais, c'est $\mathbf{L}_{2}$ (Antoine) qui sélectionne $\mathrm{L}_{1}(\mathrm{Emma})$.Ce bouleversement de rôles, est dû au parcours organisationnel des tours entre les différents participants Voir infra p.9.

78)KERBRAT-ORECCHIONI(C.),Les verbales.Approche interactionnelle et structure des conversations, T.I,p. 182.

79)Les ratés "sont imputables:

-Soit au fait que les indices sur lesquels repose l'application des règles d'alternance sont souvent flous,et peuvent donner lieu à méprise:on a alors affaire à un raté involontaire;

-Soit au fait que tout en étant d'accord sur l'interprétation des signaux émis,les différents partenaires en présence ne sont pas tous forcément disposés à s'y soumettre(...).Il y a alors violation délibérée des règles,due à une divergence d'objectifs entre $L_{1}$ et $L_{2}$ ( l'un voulant que l'autre parle qui ne le désire pas ,ou inversement)." ibid,p.172.

80) ibid,p.274.

81)Dans un polylogue"un groupe d'interlocuteurs tend à se scinder en plusieurs dyades ou couples conversationnels"

DURRER(S.),Op.Cit, p.56.

82)"La notion de statut renvoie à un ensemble de positions sociales assumées par un sujet (sexe,âge,métier,position familiale, religieuse,sociale,politique...) constituant autant d'attributs sociaux."

VION(R.), Op.Cit,p.78. 
83) KERBRAT-ORECCHIONI(C.),Les interactions

verbales.Approche interactionnelle et structure des

conversations, T.I,p.181.

84)Les déictiques ou les embrayeurs"permettent d'ancrer un énoncé dans sa situation d'énonciation ,processus qu'on nomme communément embrayage énonciatif."

MAINGUENEAU(D.),Manuel de linguistique pour les textes littéraires,Paris,Éd.Armand Colin, 2010,p.65.

Les déictiques de personne sont les pronoms personnels qui"désignent les protagonistes du dialogue."ibid,p.67.

Pour les déictiques temporels et spatiaux,Voir ibid,p.66 .

85)Ce pronom correspond à un "tu pluriel"

KERBRAT-ORECCHIONI(C.),L'énonciation de la subjectivité dans le langage,Paris,Éd.Armand Colin/Masson,1997(Troisième édition),p.41.

86) ID,Les actes de langage dans le discours.Théorie et fonctionnement, p.96.

87) Ceci relève du fait que:"Le secret et le silence lui(à Emma) avait toujours semblé les meilleurs armes,elle n'était pas disposée à en changer." BOURDIN(F.),Op.Cit, p.109.

88) KERBRAT-ORECCHIONI(C.),Les interactions

verbales.Approche interactionnelle et structure des

conversations, T.I,p.202.

89)Il y a des échanges constitutés de trois interventions:initiative,réactive et évaluative:

"-Le terme d"'évaluation ne doit pas être pris ici dans son sens usuel:il désigne simplement le troisième temps de l'échange par lequel $L_{1} \quad$ (Marie-Angèle) clôt cet échange qu'il a lui-même ouvert,en signalant à $L_{2}$ qu'il a bien enregistré son intervention réactive ,et qu'il la juge satisfaisante." ibid,p.236.

90) ibid,p.174.

91)"Les modalités d'énoncé renvoient au sujet et manifestent son attitude vis-à-vis du contenu de l'énoncé."

CALAS(F.),CHARBONNEAU(D.-R.), Op.Cit, p.49. 
92)On distingue la modalité aléthique,épistémique et déontique:"(du grec ta deonta:"ce qui doit être") exprime la nécessité en termes d'obligation morale,portée par les verbes devoir ou falloir." Loc.Cit.

93) KERBRAT-ORECCHIONI(C.),Les interactions

verbales.Approche interactionnelle et structure des

conversations, T.I,p.169.

94)VION(R.), Op.Cit,p.130.

95) Loc.Cit.

96) Emma est considérée comme le chef des Soubeyrand

qui:"(...)se faisait toujours un sang d'encre pour eux.Aujourd'hui encore,bien qu'ils soient devenus adultes,ils étaient tout pour elle."

BOURDIN(F.),Op.Cit, p.15.

97) KERBRAT-ORECCHIONI(C.),Les interactions verbales.Approche interactionnelle et structure des conversations, T.I,p.84.

98)Le chevauchement est cité avant les pauses par respect de l'ordre des propriétés de tours.

99) KERBRAT-ORECCHIONI(C.),Les interactions verbales.Approche interactionnelle et structure des conversations, T.I,p.171.

100)Ces pauses"apparaissent entre deux tours(...)"ibid,p.162.

101)L'amour commun d'Antoine et de Paul pour Marine a empoisonné leur relation fraternelle:"(...)la jeune femme avait fait d'eux des rivaux, des ennemis."

BOURDIN(F.),Op.Cit, p.25.

102)La troncation correspond au"(...)cas où une intervention à prétention initiative ne donne lieu à aucune réaction,verbale ou non verbale:elle n'est tout simplement pas prise en compte par L2."

KERBRAT-ORECCHIONI(C.),Les interactions verbales.Approche interactionnelle et structure des conversations, T.I,p.235.

103) ID,Les actes de langage dans le discours.Théorie et fonctionnement, p.62. 
104) BOURDIN(F.),Op.Cit, p.12.

105) Dans "(...) la dernière lettre anonyme qui lui(à Herrero) avait été

adressée.Le corbeau y faisait allusion à un véhicule tout-terrain et suggérait de confier celui-ci au laboratoire scientifique de la police.Sept ans plus tôt,Antoine possédait déjà ce 4x4." ibid,p.164.

Comme Herrero faisait confiance à "(...)son mystérieux correspondant dont toutes les informations se révélaient justes. ",il a ordonné une perquisition au domicile d'Antoine qui était arrêté.ibid,p.165.

106) ibid,pp.192-194.

107)VION(R.), Op.Cit,p.129.

108) Loc.Cit.

109) Loc.Cit.

110) Cet appellatif a "pour fonction de thématiser le rôle social" de Claire.

TISSET(C.),Analyse linguistique de la narration,Paris,Éd.Sedes,2000,p.28.

111)"L'anaphore se définit par toute reprise d'un élément précédemment évoqué que l'on appelle antécédent." ibid,p.32.

112) Ce déictique de personne ne représente pas"(...)une amplification de la personne."

MAINGUENEAU(D.),Op.Cit,p.67.

Il est"une sorte de barrière psychologique que l'on dresse autour de son propre territoire :en tenant ainsi l'autre à distance,et en lui refusant les marques de l'intimité(...)".

KERBRAT-ORECCHIONI(C.),Les interactions verbales, TII,Paris,Éd.Armand Colin,1992,P.62.

113) ID, Les interactions verbales.Approche interactionnelle et structure des conversations, T.I,p.89.

114) ibid,p.171.

115) ibid,p.180 .

116) ibid,p.164. 
117) Les ANL(Les actes non langagiers) renvoient aux "gestes,mimiques et autres productions corporelles."

KERBRAT-ORECCHIONI(C.),Les actes de langage dans le discours. Théorie et fonctionnement, p.150.

Ces ANL accompagnent les énoncés ou les "AL (les actes de langage)" Loc.Cit.

118) ibid,p.154.

119) Loc.Cit.

120) Loc.Cit.

Les rôles joués par les ANL proviennent de la nature de la communication qui "est à la fois multicanale et plurisémiotique" ibid,p.150.

En d'autres termes,au cours de la communication"Les ANL fonctionnent en symbiose avec les AL" ibid,p.151.

121) Loc.Cit.

122)Outre la valeur interrogative,les exclamations expriment l'étonnement du juge face au silence d'Antoine qui lui a tenu tête lors de son arrestation: "-Je n'ai pas le seul $4 x 4 d u$ département!s'insurgea Antoine" contre Herrero.

BOURDIN(F.),Op.Cit, p.164.

123)Le cadre interactif est défini"comme la nature du rapport social établi d'entrée par et dans la situation (communicative),rapport qui se maintient jusqu'au terme de l'interaction."

VION(R.), Op.Cit,p.110.

KERBRAT-ORECCHIONI(C.) attire l'attention sur le rôle déterminant du type de la situation communicative dans la précision de la valeur illocutoire des énoncés:"en situation d'interview par exemple,la plupart des énoncés produits par l'intervieweur,qu'ils se présentent ou non comme des questions auront tendance à être pris pour ce qu'ils sont en effet: des moyens plus ou moins directs ou détournés d'obtenir des informations de,et sur,l'interviewé."

KERBRAT-ORECCHIONI(C.),Les actes de langage dans le discours. Théorie et fonctionnement, p.90. 
124) ibid,p.86.

125)"Dire qu'une intervention donnée possède un caractère plus ou moins contraignant sur la suite,c'est donc admettre qu'elle crée une attente plus ou moins forte d'un enchaînement réactif."

KERBRAT-ORECCHIONI(C.), Les interactions verbales.Approche interactionnelle et structure des conversations, T.I,p.203.

126) ibid,p.235.

127) BERTHELOT(F.),Op.Cit, p.201.

128) Loc.Cit.

129) Ce qui empêche Antoine de parler c'est le respect du'pacte qui liait les Soubeyrand entre eux.Seuls Emma,ses quatre enfants et Marie-Angèle partageaient le secret qui avait failli les détruire et qui les empoisonnait toujours."

BOURDIN(F.),Op.Cit, p.40.

130)Voir supra .p.12.

131)L'innocence d'Antoine a été prouvée grâce à Sophie qui a dévoilé le secret des Soubeyrand au juge.La nuit de sa disparition,Labaume a traîné Sophie de force dans une cabane pour la violer:

"Je criais comme une folle, il m'a giflée deux ou trois fois mais je ne voulais pas me taire,c'est seulement quand il m'a jetée par terre que j'ai eu le souffle coupé.(...).J'ai continué à crier, j'ai crié tout le temps que ça a duré,(...).Lui,il parlait beaucoup...(...).Il sentait l'alcool et il me faisait très mal.J'ai crié longtemps,jusqu'à ce qu'Antoine lui tombe dessus.(...).Antoine a compris ,il a foncé.Quand il a surgi,Labaume se rhabillait.Moi j'étais sonnée, choquée...(...).Antoine avait l'air d'un fou,il a envoyé dinguer Labaume contre un des murs...Là,il s'est comme...empalé sur un gros morceau de métal qui dépassait,entre deux pierres,et il est tombé d'un bloc.J'ai dî m'évanouir parce que,après, tout est flou...Ma mère est arrivée,avec mes deux autres frères.Je ne parvenais pas à leur parler."

BOURDIN(F.),Op.Cit, pp.199-200.

Face à cet homicide involontaire, Emma "avait délibérément convaincu ses fils de cacher le corps de Labaume, maquillant ainsi en disparition ce qui était un accident et non pas un crime. Pour avoir défendu sa soeur,Antoine ne méritait pas la prison,et qu'il ait 
voulu flanquer une correction au violeur était somme toute légitime.Mais il n'avait pas souhaité sa mort et seule la malchance avait tué Labaume cette nuit-là.Quant à porter l'affaire sur la place publique,non,Emma n'avait pas pu s'y résoudre;devant Sophie en état de choc,elle n'avait songé qu'à la préserver."ibid,p.201.

La police a découvert que la personne qui envoyait les lettres anonymes à Emma et au juge était Raphaël,le témoin secret du viol de Sophie.Fils du maire de Cucuron,Raphaël était également l'ami d'enfance de Sophie dont il était éperdument amoureux:

"Elle l'attirait toujours comme un aimant,il n'y pouvait rien,ne cherchait même pas à lutter.Et jamais il ne comprendrait pourquoi elle avait épousé Arnaud,ce sinistre avocat trop âgé et trop content de lui.Sophie avec ce type,c'était comme offrir de la confiture aux cochons." ibid,p.126.

Mais ,personne ne connaissait pourquoi il envoyait ces lettres:

"Raphaël voulait-iI que Sophie n'oublie jamais cette nuit de juillet où elle s'était fait violer?Tant qu'elle allait mal,qu'elle restait fragile,elle avait besoin de lui.Qu'avait-il pu échafauder,dans sa tête de malade?Pourquoi dès le retour d'Antoine,avait-il écrit au juge,désignant le puits de Garbaud qui contenait la dépouille de Labaume?Parce qu'Antoine en tuant Labaume l'avait privé de sa vengeance et qu'il souhaitait le punir? Enfin ,s'il était fou amoureux de Sophie,comment avait-il pu supporter de voir Labaume sur elle?Un fantasme qu'il était incapable de concrétiser seul?'ibid,p.277. 


\section{Bibliographie}

\section{I.Corpus:}

\section{BOURDIN(F.):}

- Un été de canicule, Paris, Éd.Belfond,2003.

\section{II.L'oeuvre de BOURDIN(F.):}

** Romans:

-Les Soleils mouillés, Paris, Éd. Julliard, 1972.

-De vagues herbes jaunes, Paris,Éd. Julliard, 1973(adapté par Josée Dayan).

-Sang et Or, Paris, Éd. de la Table Ronde,1991.

-Mano à Mano, Paris, Éd. Denoël, 1991 (adapté pour France 3 par Victor Lanoux).

-Les Vendanges de juillet,Paris, Éd. Belfond, 1994.

-Juillet en hiver, Paris, Éd. Belfond, 1995.

-Terre Indigo,Paris,Éd.J'ai Lu ,1996 (adapté à la télévision par Victor Lanoux).

-La Camarguaise, Paris, Éd. Belfond,1996.

-Comme un frère, Paris, Éd. Belfond,1997.

-Nom de jeune fille, Paris, Éd. Belfond,1999.

-L'Homme de leur vie,Paris, Éd.Belfond,2000.

-Le Secret de Clara, Paris, Éd. Belfond, 2000.

-L'Héritage de Clara, Paris,Éd. Belfond, 2001.

-L'Héritier des Beaulieu,Paris, Éd. Belfond, 2003.

-Les Années passion : Le roman d'une femme libre, Paris, Éd. Belfond,2004.

-Un mariage d'amour, Paris, Éd. Belfond, 2004.

-La Maison des Aravis,Paris,Éd. Belfond,2004. 
-Choix d'une femme libre, Paris, Éd. Belfond, 2005.

-Les Sirènes de Saint-Malo,Paris, Éd. Belfond, 2006.

-Objet de toutes les convoitises, Paris, Éd. Belfond, 2006.

-Rendez-vous à Kerloc'h, Paris, Éd. Belfond, 2006

-Les Bois de Battandière,Paris, Éd. Belfond, 2007.

-Une passion fauve, Paris, Éd. Belfond, 2007.

-Une nouvelle vie, Paris, Éd. Belfond, 2008.

-Bérill ou la passion en héritage, Paris, Éd. Belfond, 2007.

-L'Inconnue de Peyrolles, Paris,Éd. Belfond, 2008.

-Un cadeau inespéré, Paris, Éd. Belfond, 2008.

-Une nouvelle vie, Paris, Éd. Belfond, 2008.

-Dans le silence de l'aube, Paris, Éd. Belfond, 2008.

-Sans regrets, Paris, Éd. Belfond, 2009.

-Un soupçon d'interdit, Paris,Éd. France Loisirs, 2009.

-D'espoir et de promesse, Paris, Éd. Belfond, 2010.

-Berill, Clara, Lucrèce..., Paris,Éd.Omnibus,2010.

-Les Landes en héritage, tome 1 : Des grondements de l'océan, Paris, Éd. Belfond, 2011.

-Le Testament d'Ariane, Paris, Éd. Belfond, 2011.

-Dans les pas d'Ariane, Paris, Éd. Belfond, 2011.

-Serment d'automne, Paris, Éd. Belfond, 2012.

-Maisons et secrets, Paris, Éd. Omnibus, 2012.

-BM Blues, Paris, Éd. Belfond, 2012.

-D'eau et de feu, Paris, Éd. Belfond, 2013. 
-Galop d'essai, Paris, Éd. Belfond, 2014.

\section{III.Ouvrages théoriques:}

**Ouvrages linguistiques:

-BANGE(P.), Analyse conversationnelle et théorie de l'action,Paris,Éd.Didier,1992 .

BAYLON(C.),MIGNOT(X.),La communication,Paris,É d.Nathan,1997.

-BERTHELOT(F.),Parole et dialogue dans le roman,Paris,Éd. Nathan Université,2001.

-CALAS(F.),CHARBONNEAU(D.-R.),Méthode du commentaire stylistique,Paris ,Éd.Nathan,2000.

-DURRER(S.),Le dialogue dans le roman,Paris,Éd.Nathan Université,1999.

-KERBRAT-ORECCHIONI(C.):

*Les interactions verbales.Approche interactionnelle et structure des conversations,T.I,Paris,Éd.Armand Colin/Masson,1998(Troisième édition).

*Les interactions verbales, TII,Paris,Éd.Armand Colin,1992.

* La Conversation, Paris,Éd. Seuil,1996.

*L'énonciation de la subjectivité dans le langage,Paris,Éd.Armand

Colin/Masson,1997(Troisième édition).

*Les actes de langage dans le discours. Théorie et fonctionnement,Paris,Éd.Nathan,2001.

-MAINGUENEAU(D.):

*Les termes clés de l'analyse du discours, Paris,Éd.Seuil, 1996. 
*Analyser les textes de communication, Paris,Éd. Nathan,2000.

*Manuel de linguistique pour les textes littéraires,Paris,Éd.Armand Colin,2010.

-PERRET(M.),l'énonciation en grammaire du texte,Paris,Éd.Nathan,1994.

-SIOUFFI(G.),RAEMDONCK(D.V),100 fiches pour comprendre la linguistique, Paris,Éd.Bréal,Rosny,1999.

-TISSET(C.),Analyse linguistique de la narration,Paris,Éd.Sedes,2000.

- TRAVERSO (V.), L'analyse des conversations, Paris,Éd. Nathan,1999.

-VION(R.), La communication verbale.Analyse des interactions, Paris,Éd.Hachette,1992.

IV.Dictionnaires:

-Petit Larousse en couleurs, Dictionnaire encyclopédique, Paris,Éd.Larousse,1991.

-Le Robert Mini.langue française et noms propres, Paris,Éd.Dictionnaires LE ROBERT,1995.

-CHARAUdEAU(P.),MAINGUENEAU(D.),

Dictionnaire d'analyse du discours, Paris,Éd.Seuil,2002.

-NEVEU(f.),Dictionnaire des sciences du langage, Paris,Éd.Armand Colin,2004.

V.Sitographie:

-"Laisse-moi terminer

Interaction et interruption .Analyse conversationnelle"

https://jyx.jyu.fi/dspace/bitstream/handle/123456789/13 684/skohonen.pdf? sequence=1 consulté le 20/1/2014. 
-"Les enjeux de l'analyse conversationnelle ou les enjeux de la conversation" consulté le 7/4/2014.

http://www.erudit.org/revue/rq1/2001/v30/n1/000517a r.html consulté le 18/2/2014

-"Les pauses et les silences dans l'interaction verbale"

http://www.persee.fr/web/revues/home/prescript/arti cle/lsoc_0181-4095_1984_num_29_1_1999 consulté le 9/3/2014.

- "Pour une analyse des tours de parole :

L'exemple de la conversation théâtrale"

http://romdoc.amu.edu.pl/Naruk.pdf consulté le. 5/2/2014.

-"Tours de langue et de parole"

http://www.interbible.org/interBible/source/coeur/20 00/coeur_000314. htm consulté le 20/1/2014.

-http://www.babelio.com/auteur/FrancoiseBourdin/15504 consulté le 18/05/2014.

http://www.pocket.fr/site/francoise_bourdin_\&181\&582 7.html consulté le 17/06/2014.

http://bibliobs.nouvelobs.com/romans/20120525.0BS65 99/francoise-bourdin-l-ecrivain-qui-vend-500-000livres-par-an-sans-faire-de-bruit.html consulté le 20/05/2014.

http://www.lejdd.fr/Culture/Livres/Actualite/FrancoiseBourdin-l-inconnue-des-best-sellers-491514 consulté le 7/06/2014. 